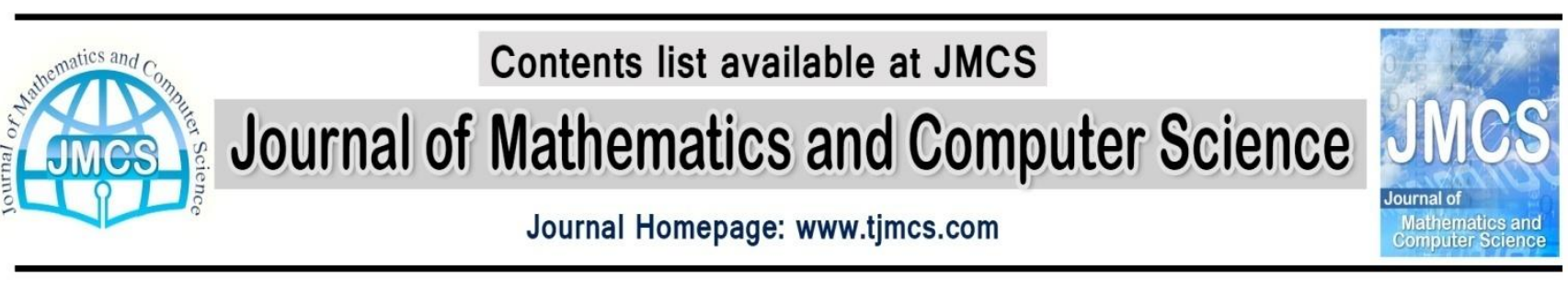

\title{
The method of GBR optimization by special parameters to decrease energy consumption in WSNs
}

Article history:

\author{
Sina Hedayati ${ }^{1}$, Arash Ghorbannia Delavar ${ }^{2}$ \\ Department of Computer, Payame Noor University, \\ PO BOX 19395-3697, Tehran, IRAN \\ hedayatisina@gmail.com ${ }^{1}$, a_ghorbannia@pnu.ac.ir²
}

Received August 2013

Accepted September 2013

Available online September 2013

\begin{abstract}
One of the fundamental for the design of routing protocols in wireless sensor networks, is energy efficiency. And because of the limitation in energy supplement, one of the most important parameters in a wireless sensor network nodes. To increase energy efficiency, The use a well-know protocol as GBR. However, there are some shortcoming in the GBR project. Firstly, to increasing elected nodes to forward the data from the source node to the sink waste to energy. Secondly, Due to the competitive nature of the algorithm, To select the most appropriate nodes to forward the data from the source node to the sink to be limited. As result, for the above problems optimization procedure discussed GBR, The main idea of this algorithm is to reduce retransmissions and attempt to save energy by taking smaller positions for to forward data in this project. This proposed scheme results in better energy efficiency than traditional GBR offers.
\end{abstract}

Keywords: Wireless sensor network, Gradient-Base Routing, Efficiency energy.

\section{Introduction}

Wireless Sensor Network (WSN) consists of a large number of nodes that have been randomly developed based on the specific circumstances. Wireless Sensor Networks are used as a technology to supply the sensitive geographical equipment in the large development area of these electronic devices [1]. The applications of Wireless Sensor Network include security detection systems, surveillance [2], 
military, target tracking, habitat monitoring [3], medical industry and its applications [4] and etc. Therefore, many articles have reviewed the issue of energy efficiency in Wireless Sensor Networks. In a wireless sensor network, the request message is broadcasted to all nodes on the network by the base station. Data is transferred to a sink for further processing by the network nodes (as sink nodes). Basically, the amount of energy consumption depends on the processing time and data transmission time among them. Thus, our purpose in this article is to reduce retransmission of data in a network and to find the more optimal way to reduce the energy consumption in nodes. Since wireless sensor networks have limited access to energy, energy consumption is one of the most important items for wireless sensor networks.

Each node independently finds its next leap into the sink and highlights a direct path for transmission that saves high energy. This scheme is used for the sensor networks which are in a restricted area. This scheme, in addition to being able to broadcast and balance a network, is used to reduce the number of transmissions in order to save energy. Many articles focus on the data routing with energy constraints. Many strategies have been proposed in the data broadcast and ability to balance network and reducing data transmissions, such Geographic Adaptive Fidelity (GAF) [5], Low Energy Adaptive Clustering Hierarchy (LEACH) [6], Threshold sensitive Energy Efficient Network protocol (TEEN) [7], and GradientBased Routing (GBR) [8] , are among the routing strategies proposed in literature. LEATH and TEEN are cluster-based routing protocols. The extra overheads of forming clusters diminish the gain in energy consumption. GAF and GBR are location-based routing protocols. In GAF, the GPS information is needed by each node to set its location which consequently increases the cost of each node. GBR is developed from the Directed Diffusion protocol[9,10]. The basic idea record the number of hops when a nod receives a message; subsequently, each node sets up its height according to the minimum number of hops. The heights' difference between a node and its neighbours forms the gradient on the corresponding link, many articles have developed GBR protocol using auxiliary techniques for data aggregation and data traffic.

\section{Related Works}

This article aims to modify GBR protocol in energy consumption. Since the proposed approach in this article is to solve problems of GBR protocol using Network coding method, [11,12] several paths are combined with routing in network coding. The sink receives its message from the source. Before the node forwards source message, it must be aware of the rout regardless the number of them. The advantage of this algorithm is that it is reliable and guarantees the energy saving. The main idea of competitive algorithm is exploiting the wireless environment, which includes a set of routes rather than a traditional path and also includes a message driving that selects and sends the message properly. In the schemes based on CBF, the main task of source node is to distribute data packets to all neighbors and then forwards data by one of its best nodes [13,14,15]. To do this, three suppression plans are presented: public suppression, regional suppression and active transmission suppression. The aim of suppression algorithm is to prevent numerous nodes and duplicate packets. 
These three mentioned schemes are to prevent duplicate packets but under the specific circumstances. Novel Message Forwarding Technique on the basis including geographic position is randomly selected through the connections among them. A receiver which is closer to the destination has high priority. As a result, the nodes closed to the destination are constantly selected and in the effect of the same energy, these nodes arrive to the end and disappear sooner. Integration routing and MAC protocol enhance throughput for transmission over a wireless and multi-hop network.[16] The performance of EXOR in a group selects the source nodes which have a list of driving positions based on the priority of closeness to the destination. The algorithm which loads the intended progress based on the priority of message forwarding is EPA that is a rate in order to obtain maximum power.

LNCS is a protocol for sensor network that is able to divide the data about location used to several non-overlapping cells for data availability. [18] SBLNC is a protocol which guarantees the security of data broadcast using linear coding network in wireless sensor networks. In [19], the authors propose an adaptive network coding approach to enhance reliability in WSNs by considering redundancy. An analytic model is then proposed to estimate the proper amount of redundancy. Further, they propose a cluster-based and distributed scheme to dynamically adjust the redundancy at each hop. However, this algorithm considers redundancy to guarantee the reliability which is not suitable for the broadcast scenario.

Network coding is forwarding data based on the slope of connections among them. Data is always sending on the shortest route. GBR is able to select the shortest route toward the sink and balance the energy in the whole rout.

\section{Proposed Algorithm}

The main idea of competitive algorithm investigated in this article is to reduce retransmission in order to decrease the energy consumption. For this purpose, it follows two main aims.

First, there are several hops states for data transmission. Second, how we can select the best node in transmission state. To meet the first aim, the energy consumption is analyzed in nodes using GBR protocol. One of the problems of this protocol that is confronting to different hops states is improved. We have tried to not merely consider the competitive algorithm given conducted investigations, but add additional parameters in selecting more appropriate nodes in order to improve energy efficiency in wireless sensitive networks; and this was our aim in this article.

The proposed algorithm applies for optimizing energy consumption through the proper selection of node and minimizing the possibility of the node selection and doesn't merely rely on competitive algorithm for forwarding the data. In next stage, when the nodes are prioritized based on the time of ACK and DACK and when the nods are sent, the source node doesn't consider merely to the first ACK and DACK received but considers to the levels of node location for data sending toward the sink that is placed at the higher priority compared to the source node. To this purpose, according to the flowchart in figure 1, we indicate the transmission of message from the source node to the sink. 
The Nodes placed in neighbor of source node send their interest message. After reaching the interest message to the receiver, the node which has received message ACK, send it to the source node. In the source node, the time of requested message and the time of message confirmation returning are calculated. If the average of these two be less than the threshold time considered, the source node put the neighbors which have this index in its destination list.

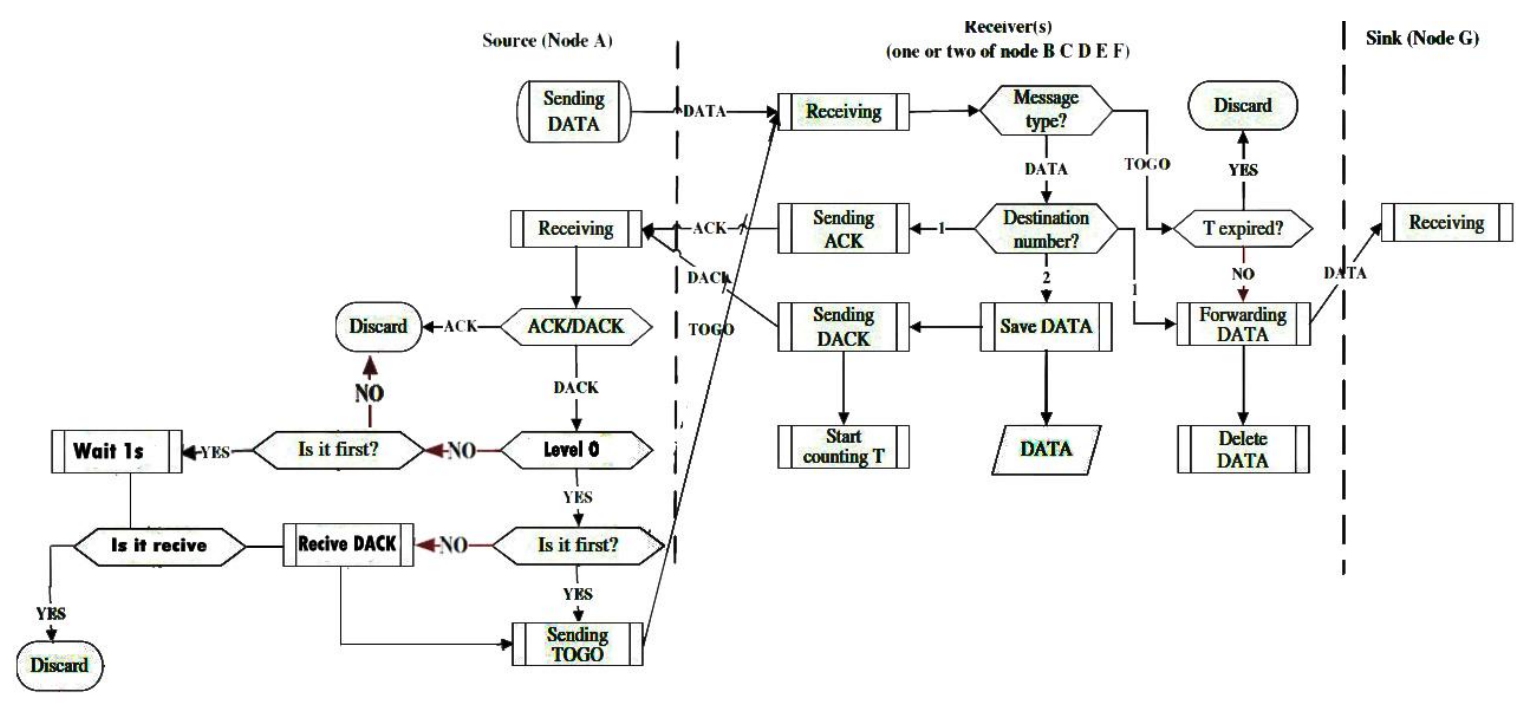

Fig1: Competitive algorithm with prioritizes nodes GBR-SE

The nodes which have the higher priority are considered as the last source. To predict the control of neighbor's nodes, there is a counter that counts the number of ACKs sent to the nodes.

\subsection{Reliability Algorithm}

In this part, we implicitly supposed that our messages reach to the destination intact. In sensor network it is important that ensure the data arrives intact to the destination in every hop. Our presented algorithm is based on GBR protocol that investigates the state of the nodes hoping in every leap. The performance is presented in figure 2 .

1- First, the sink broadcasts the interest message across the network.

2- All nodes save the number of remaining hops to the sink in their lists. When the nodes receive the interest messages from their neighbors, check and prioritize them. Only the neighbors placed in priority of message receiving that, possess the lowest number of forwarding position. 
3- The receiver of message receives the number of forward positions from destination address table.

4- The message confirmation time and the time of requesting massage are calculated.

5- If the hop position recorded in destination address be equal to one, it directs the message toward the sink.

6- If the number hop position recorded in requested message of source node is double or triple or more, it is sent to the source node of message as DACK. In source node, this message will be checked.

7- The first check for ACK and DACK is such a way that the time of receiving message from the node must be less than one.

8- If the DACK sent to a node is coplanar with source node, the intended node is selected and the message of data is sent.

1: candidates the neighbour list with the nimum

Height

2: forwarder the destination list

3: $\mathrm{T}$ waiting time $1 \mathrm{~s}$

4: generate the data message

5 : if candidates $\leq 2$ then

6: forwarders two random forwarder nodes from

candidates

7: else

8: forwarders candidates

9: end if

10: send DATA message to forwarders

11: if received a DATA

12: if the number of destination address is one then

13: send the DATA message to the sink

14: send an ACK to the sender

15: else

16: send a DACK to the sender

17: start counting $\mathrm{T}$

18: end if

19: else if received an ACK then

20: delete it

21: else if received a DACK then

22: if DACK is level0

22: if it is the first DACK for the sent DATA message

then

23: send TOGO to the sender of the DACK

24: if DACK not is level 0

25: if it is the first then

26: wait $1 \mathrm{~s}$

27:if receive DACK level 0

28: send TOGO to the sender of the DACK

29:delete it

30:Else if send TOGO DACK of first

27:else

24: end if

25: delete it

26: else if received a TOGO then

27: if $\mathrm{T}>0$ then

28: stop T

29: send the DATA message to the sink

30: end if

31: delete

32:end if 
9- If DACK is associated with coplanar node but is not of the first DACKs perceived and the receiving time is less than on, it places in higher priority for the node selection than the nodes that the time of receiving them is less than one but are not coplanar with source node.

10- If DACK is not related to coplanar node but is to the first receiving DACKs in source node, a time is selected to send TOGO to the node. To send the data to the sink, it waits for one second, if in waiting time DACK receives a message from a node placed in coplanar level of source node, it allocates the message to that node, and after receiving, it omits the final ACK.

One of the advantages of this algorithm is that it prevents the deviation of the path of message sending to the sink. Since we have a selection threshold to send data before the selection of node, we have a more appropriate selection with less possibility than the previous algorithm.

\section{Calculation of energy consumption}

Energy analysis in protocol GBR-SE begins from the calculation of the number of hop position in node. In respect to that, we only consider the energy of message transmission in this article and ignore the other energy consumers in wireless sensor networks, energy consumption for one leap in protocol GBR is highlighted as follows:

$$
E=\left(P_{T X}+P_{R X}\right) \Delta T
$$

$\Delta \mathrm{T}$ is the transmission time and is calculated from following relation:

$$
\Delta T=\frac{S_{\text {Data }}}{p} / \text { bitrate }
$$

From the relations 1 and 2, we can write:

$$
E=\frac{P_{P T}+P_{R X}}{\text { Bitrate }} \times \frac{S_{\text {Data }}}{P}
$$

Above relation is considered for a node which has only one hop position. If the number of the node hops from the source node to the sink increases, the possibility of receiving the message in receiving node is equal to:

$$
P_{n}=1-(1-P)^{n}
$$

As a result, we can consider the following relation for more than one hop:

$$
E=\frac{P_{T X}+n \times P_{R X}}{\text { Bitarte }} \times \frac{S_{\text {Data }}}{1-(1-p)^{n}} n=1,2,3,4,5
$$




\begin{tabular}{|c|c|}
\hline Avg . number of neighbours & Best coding scheme \\
\hline$N=8$ & 12 \\
\hline$N=8$ & 11 \\
\hline$N=7$ & 10 \\
\hline$N=5$ & 9 \\
\hline$N=4$ & 8 \\
\hline$N=3$ & 7 \\
\hline$N=2$ & 6 \\
\hline$N=2$ & 5 \\
\hline$N=1$ & 4 \\
\hline
\end{tabular}

Table 1: Select value $n$

In above calculations, when the hop position increases, the energy consumption also increases in sensor network. To prevent the increases in energy consumption, we select a threshold to select a node:

$$
T=T_{P}-\frac{1}{T_{A C K}+T_{R e q}}
$$

Given to the relations 3,4 , the energy consumption can be calculated from the following relation:

$$
E=\frac{P_{T X}+n \times P_{R X}}{\text { Bitarate }} \times \frac{S_{\text {Data }}}{1-(1-P)^{n}} \times \frac{1}{T}
$$

\begin{tabular}{|c|c|c|}
\hline & Parameter & value \\
\hline 1 & Ptx & s4.0 MW/S \\
\hline 2 & Prx & $r \mathrm{MV} / \mathrm{s}$ \\
\hline 3 & SDATA & $\wedge \mathrm{\wedge} \mathrm{bit/s}$ \\
\hline 4 & Bitrare & $19 r \cdots \mathrm{bit} / \mathrm{s}$ \\
\hline
\end{tabular}

Table 2: Energy consumption with the following parameters

The result show, due to value $\mathrm{n}$ is dropped high threshold. And limited to this range that help to save energy in wireless sensor network. It can see that the energy can be save if GBR-SE is used . A saving of up to $47 \%$ energy for one hop. As a result, it can be concluded that GBR-SE can save energy when the transmission in the range of ( 0.4 to 1$)$. 


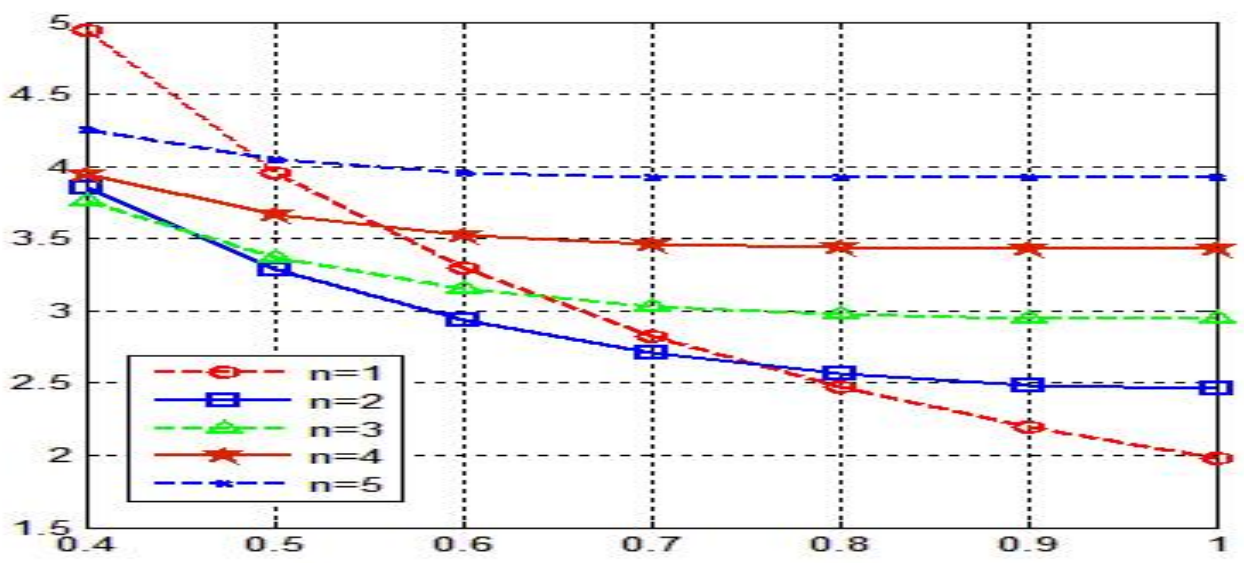

Fig 2: The optimized energy diagram

\section{Simulation and Result}

The algorithm of this article is simulated in software OMNET ++. The simulation is carried considering a wireless sensor network with a number of nodes that are randomly scattered in the environment. Density is based on the network coding scheme. All nodes are set in the network on the radio range of $180 \mathrm{~m}$. we considered the initial energy capacity of $30 \mathrm{~J}$ in OMNET ++. The power of energy consumption model Mica2 [21] presented in the charts for 500 nodes in wireless sensor networks shows the resting time of nodes and the time of sending and receiving data. As it can be seen in charts, the resting time of nodes increases in wireless sensor networks and the time of sending and receiving data are improved compared to the previous method, which saves the energy in wireless sensor network.

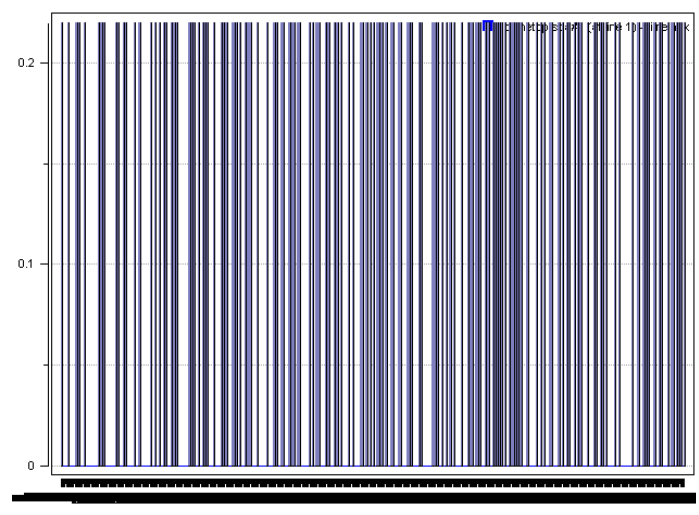

Fig3: The time of send data for wireless sensor network node 500

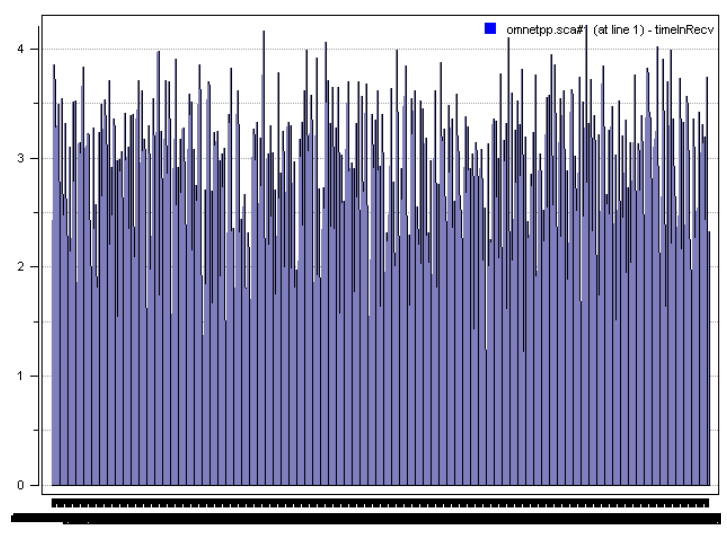

Fig4: The time of receive data for wireless sensor network nod 500 


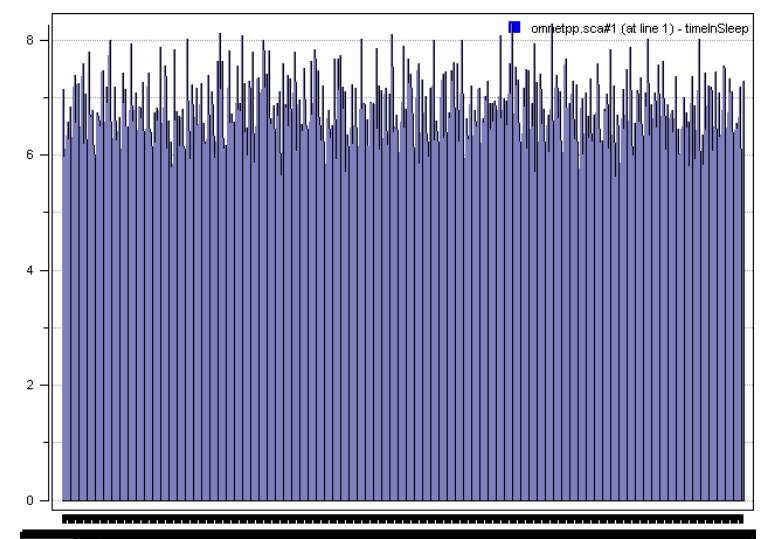

Fig5: Time sleep of nodes for wireless sensor network

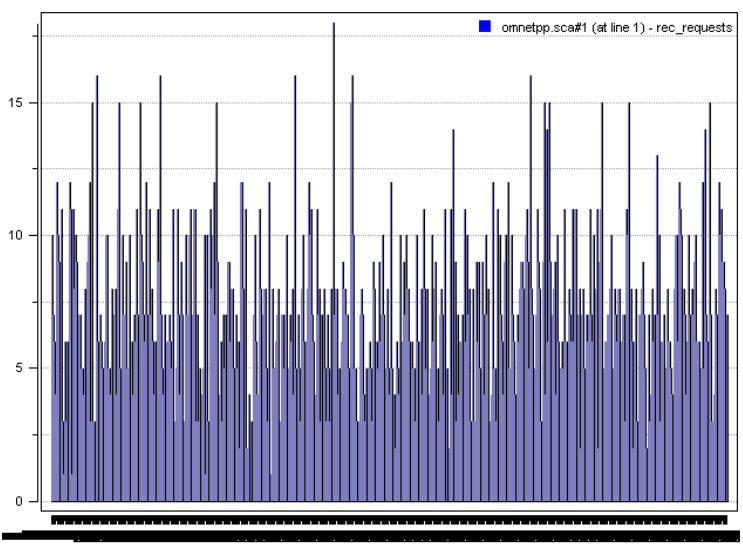

Fig6: The number of request message for each node wireless sensor network

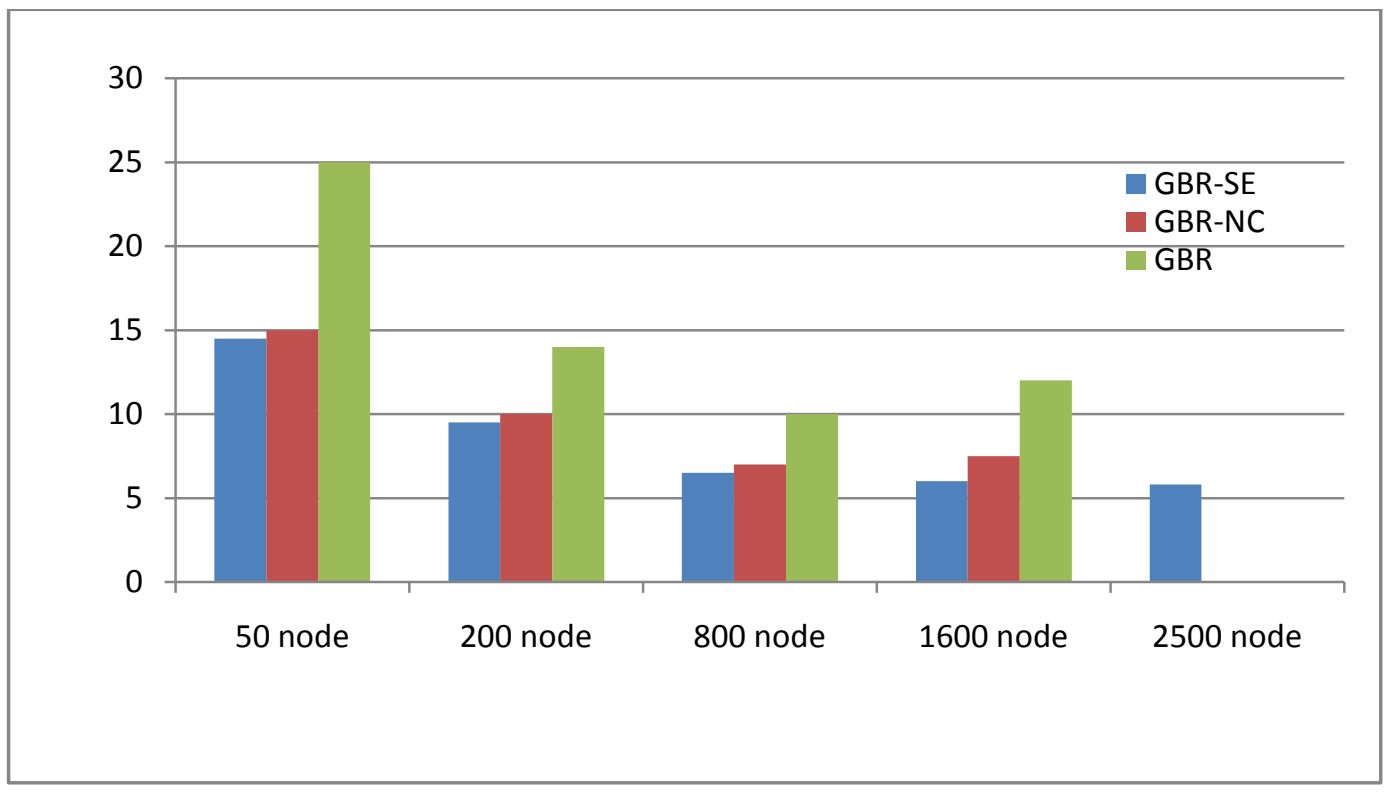

Fig7: Average energy consumption for different networks

charts provided by authors show the behavior of all nodes in a wireless sensor network. Each of which represents the behavior each nodes in the time of send data and time of receive data and time of sleep data .

According to the simulation results, we can see that this optimization algorithm more increase better than the previous algorithms have been presented in wireless sensor networks. The best selected for forwarding data and the most sleep time of node and the better selected for path for transmitting data. 
Increasing of node in the wireless sensor network on the optimization algorithm GBR-SE is not dramatically reduce the energy consumption. But these algorithms in wireless sensor networks always keeps the balance energy consumption.

\section{Conclusion}

The results of experiments show that GBR-SE algorithm is improved and it has a better efficiency energy compared with the two other algorithms, GBR and GBR-NC. Average improvement of GBR-SE as compared to GBR and GBR-C, Simulation results showed that compared with GBR around $73 \%$ energy consumption and GBR-NC around 18\% energy consumption as well as network traffic is saved by GBR$\mathrm{SE}$. The next work can focus on probability distribution for responding and concentrated processing and threshold distance

\section{Reference}

[1] Lusheng Miao, Karim Djouani, Anish Kurien, Guillaume Noel Network coding and competitive approach for gradient based routing in wireless sensor networks Ad Hoc Networks, Volume 10, Issue 6, August (2012), Pages 990-1008

[2] T. He, S. Krishnamurthy, J.A. Stankovic, T. Abdelzaher, L. Luo, R. Stoleru,T. Yan, L. Gu, Energy-efficient surveillance system using wireless sensor networks, in: Mobisys, ACM Press,( 2004), pp. 270-283.

[3] G. Tolle, J. Polastre, R. Szewczyk, D. Culler, N. Turner, K. Tu, S. Burgess, T. Dawson, P. Buonadonna, D. Gay. Hong, A macroscope in the redwoods, in: Proceedings of the 3rd International Conference on Embedded Networked Sensor Systems, SenSys '05, ACM, New York,NY, USA, (2005), pp. 51-63. Doi : http ://doi.acm.org /10.1145 /1098918 .1098925.

[4] R.K. Ganti, P. Jayachandran, T.F. Abdelzaher, J.A. Stankovic, Satire: asoftware architecture for smart attire, in: Proceedings of the 4th International Conference on Mobile Systems, Applications and Services, MobiSys'06,ACM,NewYork,NY,USA,(2006),pp.110-123

[5] Y. Xu, J. Heidemann, D. Estrin, Geography-informed energy conservation for ad hoc routing, in: Proceedings of the 7th annual international conference on Mobile computing and networking, MobiCom '01, ACM, New York, NY, USA,( 2001), pp. 70-84

[6] W. Heinzelman, A. Chandrakasan, H. Balakrishnan, Energy-efficientco mmunication protocol for wireless microsensor networks, in:Proceedings of the Hawaii Conference on System Sciences, 2000.

[7] A. Manjeshwar, D.P. Agrawal, Teen: a routing protocol for enhanced efficiency in wireless sensor networks, in: Proc. 15th Int. Parallel andDistributed Processing Symp.,( 2001), pp. 2009-2015.

[8] A.S. Izadi, A.R. Sahab, J. Vahidi, A New Mechanism for Traffic Reduction the ServiceResource Discovery Protocol in Ad-Hoc Grid Network: The Journal of Mathematics and Computer Science , 6 (2013),pp129-138

[9] J.N. Al-Karaki, A.E. Kamal, Routing techniques in wireless sensor networks: a survey, IEEE Wireless Commun. Mag. 11 (6) (2004) 6-28

[10] Hamed Azami, Milad Malekzadeh, Saeid Sanei, A New Neural Network Approach for Face Recognition based on Conjugate Gradient Algorithms and Principal Component Analysis: The Journal of Mathematics and Computer Science,(2013),pp 166 - 175

[11] L. Shan-Shan, Z. Pei-Dong, L. Xiang-Ke, C. Wei-Fang, P. Shao-Liang,Energy efficient multipath routing using network coding in wirelesssensor networks, in: T. Kunz, S. Ravi (Eds.), Ad-Hoc, Mobile, andWireless Networks, vol. 4104, Springer, Berlin/Heidelberg, (2006), pp.114-127. 
[12] Y.S.Y. Yang, C. Zhong, J. Yang, Energy efficient reliable multi-path routing using network coding for sensor network,IJCSNS Int. J.Comput. Sci. Network Secur .8(12)(2008)114-127. [13] Q. Wang, H. Hassanein, G. Takahara, Stochastic modeling of distributed, dynamic, randomized clustering protocols for wireless sensor networks, in: Proceedings of the 2004 International Conference on Parallel Processing Workshops, ICPPW '04, IEEE Computer Society, Washington, DC, USA, (2004), pp. 456-463.

[14] L. Shan-Shan, Z. Pei-Dong, L. Xiang-Ke, C. Wei-Fang, P. Shao-Liang, Energy efficient multipath routing using network coding in wireless sensor networks, in: T. Kunz, S. Ravi (Eds.), Ad-Hoc, Mobile, and Wireless Networks, vol. 4104, Springer,Berlin/Heidelberg,(2006),pp.114-127.

[15] Y.S.Y. Yang, C. Zhong, J. Yang, Energy efficient reliable multi-path routing using network coding for sensor network, IJCSNS Int. J.Comput. Sci. Network Secur.8(12)(2008)114-127

[16]Z. Xiong, W. Liu, J. Huang, W. Cheng, B. Cheng, Network coding approach for intra-cluster information exchange in sensor networks,in: Proc. VTC-2007 Fall Vehicular Technology Conf. 2007 IEEE 66th,2007,pp.164-168.

[17] E. Ayday, F. Delgosha, F. Fekri, Location-aware security services for wireless sensor networks using network coding, in: Proc. 26th IEEE Int. Conf. Computer Communications, INFOCOM 2007, IEEE, (2007), pp. 1226-1234

[18] Z. Zhu, Q. Tan, P. Zhu, Q. Zheng. Security broadcast based on linear network coding in WSN, in: Proceedings of International Computer Science and Software Engineering Conference, vol. 3, 2008, pp. 919-922

[19] T.-G. Li, C.-C. Hsu, C.-F. Chou, On reliable transmission by adaptive network coding in wireless sensor networks, in: ICC '09. IEEE International Conference on Communications, (2009), pp. 1-5. [20] J.W. Hui, D. Culler, The dynamic behavior of a data dissemination protocol for network programming at scale, in: Proceedings of the 2nd International, ACM Press, (2004), pp. 81-94. [21] V. Shnayder, M. Hempstead, B.-r. Chen, G.W. Allen, M. Welsh, Simulating the power consumption of large-scale sensor network applications, in: Proceedings of the 2nd International Conference on Embedded Networked Sensor Systems, SenSys '04, ACM, New York,NY, USA, (2004), pp. 188-200. 